\title{
Barreras para la atención de las conductas suicidas en Ciudad de México: experiencias del personal de salud en el primer nivel de atención
}

\author{
Barriers to Care of Suicidal Behavior in Mexico City: Health Personnel Experiences \\ at the First Level of Care
}

\author{
Barreiras para a atenção das condutas suicidas na Cidade do México: \\ experiências dos profissionais de saúde na atenção básica
}

Lourdes Gómez-Garcia'; Luz Arenas-Monreal2; Rosario Valdez-Santiago3; Mario Rojas-Russell4; Claudia Iveth AstudilloGarcía5; Marcela Agudelo-Botero ${ }^{6}$

1 Candidata a Doctora en Ciencias Médicas. Universidad Nacional Autónoma de México, Ciudad de México, México. lougomezg@ gmail.com. ORCID: https://orcid.org/0000-0003-4579-0010

2 Doctora en Antropología Médica. Instituto Nacional de Salud Pública, Cuernavaca, México. luz.arenas@insp.mx. ORCID: https://orcid. org/0000-0001-5071-1124

3 Doctora en Ciencias en Salud Pública. Instituto Nacional de Salud Pública, Cuernavaca, México. rosario.valdez@insp.mx. ORCid: https://orcid.org/0000-0001-8434-9805

4 Doctor en Ciencias de la Salud en Epidemiología. Universidad Nacional Autónoma de México, Ciudad de México, México. merr@ unam.mx. ORCID: https://orcid.org/0000-0001-6644-1384

5 Doctora en Ciencias de la Salud en Epidemiología. Secretaría de Salud, Ciudad de México, México. claudiaiveth.astudillo@gmail. com. ORCID: https://orcid.org/0000-0002-0895-5747

6 Doctora en Estudios de Población. Universidad Nacional Autónoma de México, Ciudad de México. magudelo@unam.mx. ORCiD: https://orcid.org/0000-0002-4489-4224

Recibido: 16/06/2021. Aprobado: 08/11/2021. Publicado: 21/12/2021

Gómez-García L, Arenas-Monreal L, Valdez-Santiago R, Rojas-Russell M, Astudillo-García CI, Agudelo-Botero M. Barreras para la atención de las conductas suicidas en Ciudad de México: experiencias del personal de salud en el primer nivel de atención. Rev. Fac. Nac. Salud Pública. 2022;40(1):e346540. Dor: https://doi.org/10.17533/udea.rfnsp.e346540

\section{Resumen}

Objetivo: Identificar las barreras que existen para la atención de las conductas suicidas, desde la perspectiva de las/los profesionales de la salud mental del primer nivel de atención de la Ciudad de México. Metodología: Estudio cualitativo exploratorio, basado en 35 entrevistas semiestructuradas, dirigidas a personal de salud de dos unidades de salud mental de referencia nacional. El análisis de la información se hizo mediante el Framework Analysis. Resultados: Las/los participantes consideraron que las conductas suicidas no han sido definidas como una prioridad dentro de los trastornos de salud mental. La ausencia de políticas públicas, la sobrecarga de trabajo, la falta de seguimiento a las/los pacientes, entre otras, fueron identificadas como barreras para una atención adecuada y oportuna. Conclusiones: Los programas de prevención de las conductas suicidas deben tomar en cuenta el contexto socioeconómico de la población y las características 
de los servicios de salud, así como las necesidades de las personas prestadoras de servicios de salud. Se requiere ampliar el entrenamiento profesional y mejorar el sistema de referencia y contrarreferencia entre los distintos niveles de atención.
-Palabras clave: acceso a los servicios de salud, Ciudad de México, primer nivel de atención, proveedores de servicios de salud, salud mental, suicidios.

\begin{abstract}
Objective: To identify the existing barriers to the care of suicidal behavior from the perspective of mental health professionals at the first level of care in Mexico City. Methodology: Qualitative exploratory study based on 35 semi-structured interviews conducted on health personnel from two national reference mental health facilities. The data were analyzed with Framework Analysis. Results: The participants considered that suicidal behavior has not been prioritized among mental health disorders. The absence of public policies, work overload, lack of patient follow-up, among others, were identified as barriers
\end{abstract}

to adequate and timely care. Conclusions: Suicidal behavior prevention programs should consider the socioeconomic context of the population, the characteristics of health services, and the needs of health care providers. Professional training should be enhanced, and the referral and counter-referral system across levels of care should be improved.

Keywords: access to health services, Mexico City, first level of care, health care providers, mental health, suicides

\section{Resumo}

Objetivo: Identificar as barreiras existentes para a atenção das condutas suicidas, desde a perspectiva dos profissionais de saúde mental de atenção básica da Cidade do México. Metodologia: Estudo qualitativo exploratório, baseado em 35 entrevistas semiestruturadas, dirigidas a profissionais de saúde de duas unidades de saúde mental de referência nacional. A análise da informação foi feita através do Framework Analysis. Resultados: Os participantes consideraram que as condutas suicidas não têm sido definidas como uma prioridade dentro dos transtornos de saúde mental. A ausência de políticas públicas, a sobrecarga de trabalho, a falta de seguimento aos pacientes, entre outras, foram identificadas como barreiras para uma atenção adequada e oportuna. Conclusões: Os programas de prevenção das condutas suicidas devem considerar 0 contexto socioeconômico da população e as características dos serviços de saúde, além das necessidades dos profissionais de saúde. É necessário ampliar o treinamento profissional e melhorar o sistema de referência e contrarreferência entre os diferentes níveis de atenção.

---------Palavras-chave: Acesso aos serviços de saúde; Cidade do México; Atenção básica de saúde; Profissionais de saúde; Saúde mental; Suicídios

\section{Introducción}

Las conductas suicidas se definen como "el comportamiento que deliberadamente causa lesiones o posibles lesiones a quien ejecuta la acción" [1, p. 21]; incluyen la ideación, la planeación y el intento suicida [1]. Entre 1990 y 2019, en el mundo, la tasa de mortalidad — para todas las edades - por suicidios disminuyó en $29 \%$, mientras que, en México, dicha tasa aumentó 97,6\%, pasando de 3,2 a 6,3 suicidios por cada 100000 habitantes en el mismo periodo [2].

En México, el suicidio es la principal causa de muerte en las personas entre 15 y 49 años [2]. De acuerdo con datos de la Encuesta Nacional de Salud y Nutrición 2018-2019 (ENSANut), se estimó una prevalencia de intento e ideación suicida en adolescentes de 3,9 y $5,1 \%$, respectivamente [3]. Por otro lado, se ha documentado que entre los factores de riesgo para presentar conductas suicidas en esta población se encuentran el consumo de sustancias, el ser víctima de violencia, la depresión y los trastornos de la conducta [4].

Las conductas suicidas no solo son un problema social; también involucran a los servicios de salud. Se sabe que la atención de la salud mental en los servicios de atención primaria ofrece una oportunidad para la intervención temprana de las conductas suicidas [5]; sin embargo, en el país se han identificado obstáculos para prestar estos servicios en unidades del primer nivel de atención [6-8]. Algunas de las barreras más frecuentes son la deficiente conducción y función de la Secretaría de Salud en salud mental, la falta de una estrategia nacional, el escaso presupuesto y la ausencia de sistematización de casos para su detección y tratamiento [9]. 
Asimismo, se ha evidenciado que la mayoría de los intentos de suicidio ocurre antes de contactar con algún proveedor de servicios de salud [10]. Los datos de encuestas de salud mental muestran que alrededor del 41 $\%$ de los adultos y el $17 \%$ de los adolescentes en México buscaron tratamiento en los 12 meses previos a un evento suicida [11]. En cambio, a nivel nacional, tan solo 8,8 $\%$ de los adolescentes que intentaron suicidarse alguna vez en la vida recibieron posteriormente atención médica o psiquiátrica [12]. De ahí que las/los profesionales de la salud en el primer nivel de atención son actores clave para efectuar estrategias de promoción, prevención y tratamiento de las conductas suicidas [13].

Aunque en México las conductas suicidas se han estudiado ampliamente desde un punto de vista epidemiológico [14], pocos estudios se han centrado en conocer el rol de los servicios de salud en el abordaje del suicidio y, en particular, sobre los obstáculos que enfrenta el personal de salud para la atención de las conductas suicidas [15]. Por lo tanto, el objetivo de este estudio fue identificar las barreras que existen para la atención de las conductas suicidas, desde la perspectiva del personal de salud mental del primer nivel de atención de la Ciudad de México.

\section{Metodología}

\section{Racionalidad del estudio}

El interés por analizar las barreras que existen para la atención de las conductas suicidas en el primer nivel de atención surgió de los hallazgos de un estudio previo, realizado en el país, donde se encontró que los adolescentes con conductas suicidas enfrentan obstáculos para acceder a los servicios de salud del primer nivel de atención. Entre estos obstáculos se describieron, por ejemplo, el poco tiempo destinado a las consultas, los horarios de atención disponibles que se traslapan con los horarios escolares, así como la alta rotación del personal de salud [16]. Este análisis, además, planteó la necesidad de explorar con mayor profundidad estas barreras, considerando distintos actores, entre ellos, los prestadores de servicios de salud. A partir de esto, la autora principal, Lourdes Gómez-García, decidió desarrollar su tesis doctoral sobre este último punto.

El equipo de investigación involucrado en este estudio tiene una formación multidisciplinaria, a nivel de posgrado, en áreas relacionadas con la salud, como epidemiología, sistemas de salud, antropología médica, estudios de población y salud pública, lo cual permitió abordar el tema desde distintas perspectivas que enriquecieron el análisis.

Para la estructuración de este artículo se siguieron los criterios indicados en los Standards for reporting qualitative research (Estándares para el reporte de investigación cualitativa) [17].

\section{Diseño de estudio}

Estudio cualitativo, de tipo exploratorio. Las barreras para la atención de las conductas suicidas se definieron como los obstáculos que enfrenta el personal de salud para la provisión de servicios, los cuales pueden ser de estructura normativa y legislativa, de organización de los servicios de salud y de características individuales/ profesionales. La presencia de obstáculos, en cualquiera de estas dimensiones, limita la posibilidad de lograr cobertura efectiva de los servicios de salud, además de impactar la calidad de la atención [18-20].

El estudio se realizó en la Ciudad de México, ya que ahí se concentra la mayor oferta de servicios de salud mental del país [21]. En el primer nivel de atención se cuenta con cuatro unidades especializadas en salud mental de referencia nacional, tres de ellas con enfoque de atención comunitaria. Para el presente estudio, se eligieron dos de estas unidades por conveniencia, con el apoyo de la Secretaría de Salud, las cuales se encuentran ubicadas en zonas caracterizadas por tener niveles de pobreza elevados [22].

La población de estudio incluyó al personal de salud, directivo y operativo, adscrito a las unidades -médicos/as, psiquiatras, psicólogos/as, enfermeros/ras y trabajadores/as sociales-; se procuró variabilidad en el perfil profesional, para incluir diferentes perspectivas de la atención de las conductas suicidas. La selección de las/los participantes se efectuó por muestreo selectivo y, para su inclusión, se buscó que las/los participantes tuvieran experiencia en la atención de las/los pacientes con conductas suicidas; se excluyó a personal de salud en formación.

La invitación a participar se hizo cara a cara, con apoyo de las/los coordinadoras/es de área y la dirección de enseñanza de cada unidad. Se obtuvieron 35 entrevistas, mientras que cuatro profesionales de la salud decidieron no participar en el estudio.

Se realizaron entrevistas semiestructuradas, empleando una guía de entrevista que se diseñó con el objetivo de identificar las barreras sobre la estructura normativa, la organización de los servicios y la experiencia de las personas vinculadas con la atención de las conductas suicidas. Todas las entrevistas se llevaron a cabo en el lugar de trabajo de las personas participantes, en el horario elegido por ellas y manteniendo la confidencialidad. La recolección de información se hizo entre enero y marzo del 2020 - previo a declararse las medidas de confinamiento por la pandemia por el virus del síndrome respiratorio agudo severo tipo-2 (SARS-CoV-2) en México-. La duración promedio de las entrevistas fue de 30 a 45 minutos. 


\section{Análisis}

Las entrevistas fueron audiograbadas digitalmente y se transcribieron literalmente en Word. Se realizó un análisis temático, empleando la metodología del Framework Analysis. Esta metodología permite identificar puntos en común y relaciones entre los diferentes datos estudiados, con lo que se puede concluir, describir o explicar un fenómeno, agrupando la información en función de temas [23].

El método siguió las siguientes etapas:

1. Se definieron temas y códigos con base en la revisión de la literatura sobre el proceso de atención de la salud y las barreras sobre conductas suicidas [1820,24-26] desde la perspectiva del personal de salud.

2. Mediante una primera lectura de las entrevistas, las autoras y el autor eligieron aleatoriamente cinco en- trevistas para, de manera independiente, identificar temas, subtemas y códigos.

3. El equipo de investigación definió los temas, subtemas y códigos finales, incluyendo códigos emergentes. La información se organizó en cuatro ejes: a) lineamientos para la atención de las conductas suicidas; b) operatividad de los servicios de salud mental; c) disponibilidad de recursos materiales y humanos, y d) experiencias de las/los proveedoras/res de servicios alrededor de las conductas suicidas.

4. Finalmente, el equipo de investigación consensuó la información de cada tema y subtema, siguiendo un análisis iterativo de datos, tanto de modo inductivo como deductivo, hasta encontrar el punto de saturación teórica, es decir, cuando la información com-

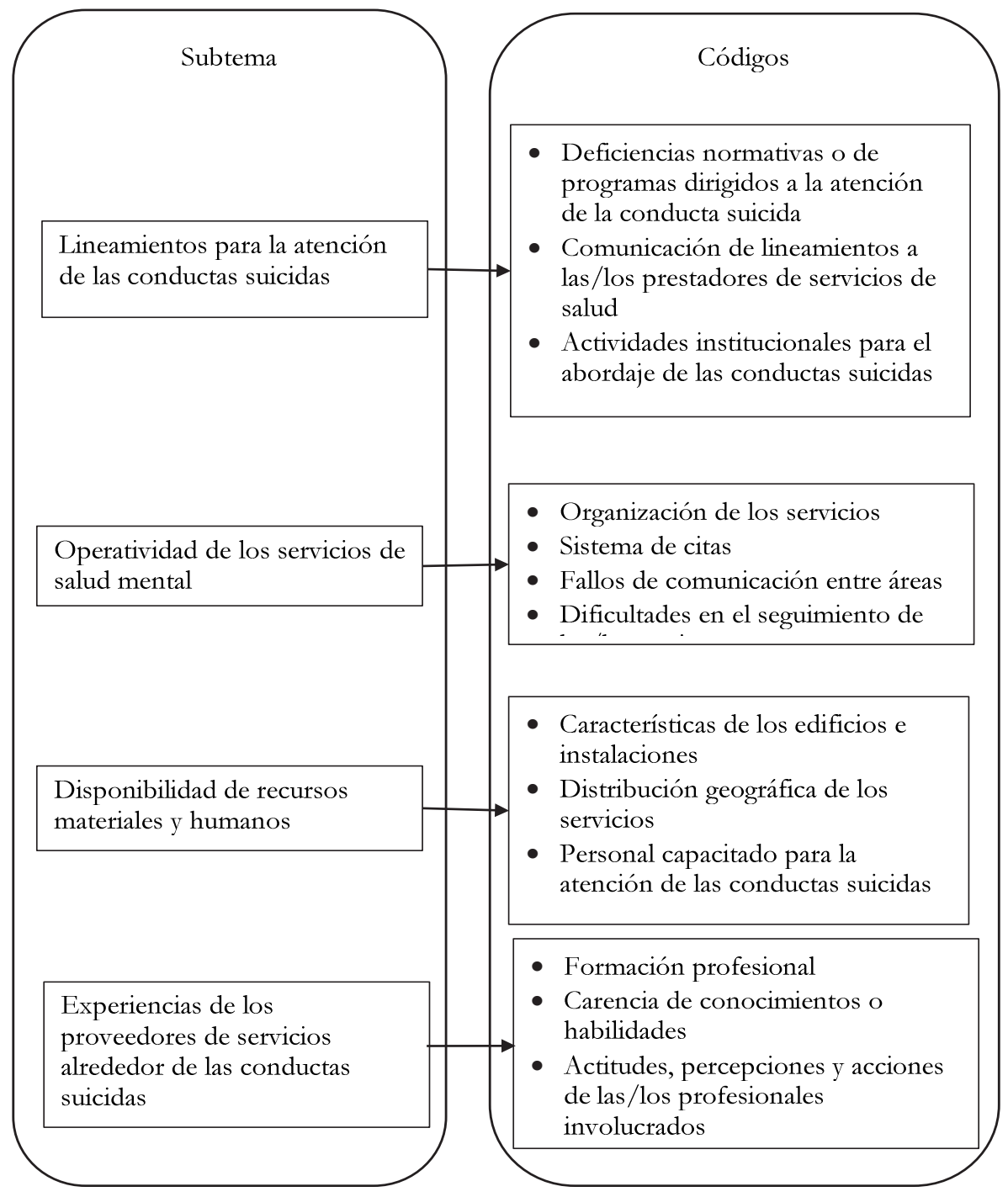

Figura 1. Árbol de codificación de las entrevistas

Fuente: Elaboración propia, con base en datos de campo. 
pilada no agregó información nueva de los temas y subtemas. Después de acordar la asignación de códigos colectivamente, se codificó la información con el apoyo del software Atlas-ti ${ }^{\circledR} 8.4 .3$ [27]. La Figura 1 presenta, de manera esquemática, un ejemplo de cómo se llevó a cabo la codificación de las entrevistas.

\section{Consideraciones éticas}

El proyecto fue revisado y aprobado por la Comisión de Ética e Investigación del Programa de Posgrado en Ciencias Médicas, Odontológicas y de la Salud de la Universidad Nacional Autónoma de México (oficio de aprobación PMDCMOS/CEI/006/2019).
La participación fue voluntaria y se solicitó la firma de un consentimiento informado escrito, previo a cada entrevista.

Para garantizar la privacidad y la confidencialidad de la información aquí presentada, a cada participante se le asignó un registro alfanumérico.

\section{Resultados}

Los datos sociodemográficos y profesionales de la población de estudio se muestran en la Tabla 1 .

Los resultados se ordenaron en torno a cuatro temas, cada uno con sus respectivos subtemas, los cuales son presentados a continuación.

Tabla 1. Características sociodemográficas de las/los participantes

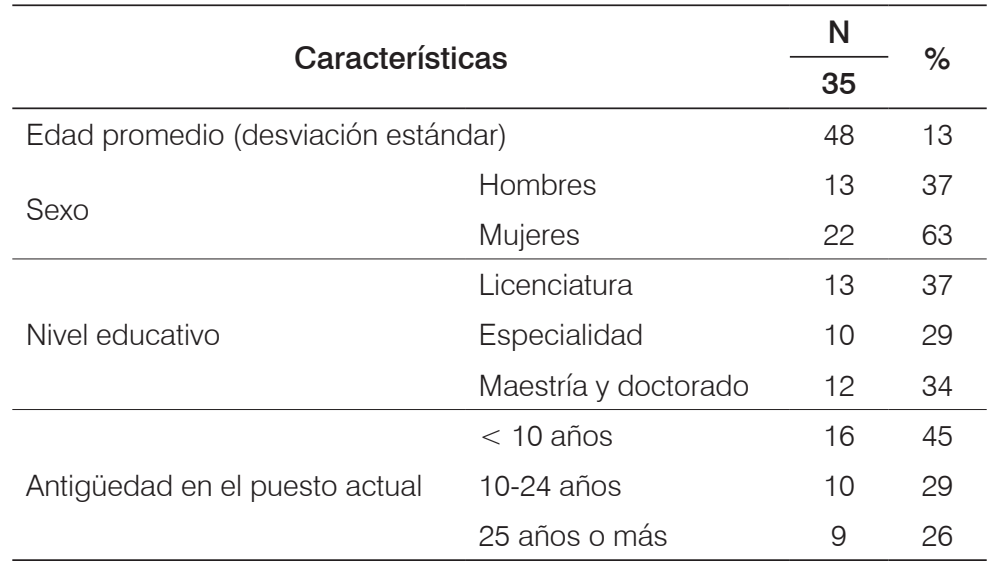

Fuente: elaboración propia, con base en datos de campo.

Tema 1. Lineamientos para la atención de las conductas suicidas

Las conductas suicidas en la agenda de salud. Las/los participantes no identificaron alguna institución o ente rector que dirija las acciones para la prevención del suicidio. Además, consideraron que la detección de conductas suicidas tiene menor importancia en los servicios de salud mental, debido a que entran en competencia con otros padecimientos como los trastornos de la conducta o las adicciones.

En la experiencia de las/los participantes, la falta de priorización de las conductas suicidas se refleja en el presupuesto insuficiente, la falta de programas específicos o la carencia de campañas de comunicación social enfocadas a este problema de salud.

Aunque mencionaron no conocer ninguna política para el abordaje de conductas suicidas y sus consecuencias, perciben que ha habido un creciente interés de las/ los tomadores de decisiones por este tema en particular.
[...] Pues, no considero que lo tomen tan prioritario [al suicidio] como otras enfermedades más físicas, como podría ser cáncer, enfermedades terminales. Creo que a la psiquiatría la han relegado siempre un poco más que a las demás disciplinas del área médica o las otras especialidades, pero que sí es de mucha importancia (oP-19-M48).

Ausencia de protocolos institucionales. Las/los participantes señalaron que no cuentan con protocolos institucionales que guíen las acciones de atención de las conductas suicidas. Indican que el único proceso establecido es la derivación de las/los pacientes con conductas suicidas de alto riesgo a hospitales de tercer nivel para su atención. Mencionan que se efectúan actividades de prevención a las conductas suicidas de forma esporádica, en fechas específicas como, por ejemplo, el Día Mundial de la Prevención del Suicidio, que se celebra cada 10 de septiembre, por lo que estas actividades no tienen continuidad. 
A nivel nacional, no [hay un programa para la prevención del suicidio]. Aquí las políticas que se usan internamente es que si lo detectamos [conductas suicidas], lo trabajemos como urgencia y lo mandemos a canalizar de manera urgente [al hospital psiquiátrico] (op-26-H31).

\section{Tema 2. Operatividad de los servicios de salud mental}

Trámites administrativos. La burocracia para acceder a los servicios de salud mental, como la solicitud de "fichas" o turnos para la obtención de una consulta — las cuales están restringidas por día-, el pago por las consultas y la presentación de documentación (como identificación y comprobante de domicilio) son barreras para la atención de las conductas suicidas. Las/los participantes sostienen que esto repercute en que algunas personas desistan en la búsqueda de atención, o que no lleguen a recibir un diagnóstico.

[Deberíamos] darles prioridad [a los pacientes con conductas suicidas], en el sentido de que si nosotros los detectamos, brindar[les] una primera atención sin esperar retribución económica, nada más para ver si se va a poder o no se va a poder trabajar con el paciente, porque a veces estamos esperando que hagan el pago, que no trae ficha, o esto (op-26-H31).

Saturación de servicios. Las unidades donde se realizó este estudio se caracterizan por la creciente demanda y saturación de los servicios que se ofertan. Estas son de referencia nacional, por lo que su población usuaria proviene de la Ciudad de México y otros estados del país.

Las/los participantes dijeron disponer de poco tiempo para las consultas o las consejerías, debido a horarios restringidos y a que deben completar una serie de formatos relacionados con la atención, además de cumplir con actividades administrativas.

Desgraciadamente, el espacio y los tiempos [de consulta] son muy cortos, por la demanda de gente. Para asesorar [a los pacientes con conductas suicidas], tendríamos que tener todavía más tiempo para dar mayores indicaciones (OP-19-M48).

Tiempos de espera y continuidad de la atención. Solo una de las unidades tiene atención en el turno vespertino, por lo que la mayor parte de los horarios de consulta interfiere con actividades escolares o laborales de las/los pacientes; además, es poco probable que estas/os puedan elegir el día y la hora de las consultas.

Para que las/los pacientes accedan a una atención psicológica o psiquiátrica, primero pasan por diversos filtros, con trabajo social, enfermería o medicina general; por lo tanto, deben acudir a más de una cita antes de que se aborde la necesidad relacionada con las conductas suicidas.
También encuentran como una limitante la falta de continuidad de la atención médica, pues el lapso entre consultas puede ser de 1 a 3 meses. Según las/los participantes, estos tiempos no favorecen el seguimiento a la atención de las conductas suicidas, ya que las/los pacientes pueden presentar un intento de suicidio antes de la siguiente cita.

La estructura del sistema no permite que veamos a los pacientes cada ocho días, cada semana, sino es cada mes. Entonces, no hay un buen seguimiento para detectar necesidades del paciente propiamente, porque están muy desfasadas las citas. [...] O sea, se les atiende en el momento, pero durante quince días o hasta un mes, el paciente puede volver a incurrir en el suicidio (OP-10-M34).

Sistema de referencia y contrarreferencia. En las unidades de salud no se cuenta con seguimiento a las referencias y contrarreferencias de los pacientes con conductas suicidas a otros niveles de atención. Cuando las/ los pacientes presentan conductas suicidas de alto riesgo, se remiten a un hospital psiquiátrico, donde reciben tratamiento farmacológico y terapéutico hasta que se determina que el riesgo ha disminuido. Al ser egresados, son contrarreferidos a la unidad de atención ambulatoria más cercana al domicilio de la/el paciente, para continuar con la atención; sin embargo, este no es un seguimiento transversal a todo el proceso de atención médica.

\begin{abstract}
En la atención, sabemos que llega un paciente aquí con intento suicida; bueno, los médicos valoran si todavía pueden estar en casa, bajo monitoreo y supervisión. Pero si su intento fue letal, lo canalizan al hospital y ya el hospital lo ve; pero cuando ellos regresan para su seguimiento, ahí es donde nos ha faltado tener ese monitoreo, cuántos pacientes de seguimiento por suicidio tenemos, por ejemplo (OP-21-M49).
\end{abstract}

\section{Tema 3. Disponibilidad de recursos materiales y humanos}

Infraestructura y materiales. Para las/los entrevistados, la infraestructura resulta insuficiente para la demanda de servicios y para ampliar los servicios preventivos, promover grupos de supervivientes de suicidio o terapias grupales, entre otros. Asimismo, se menciona la poca disponibilidad de medicamentos psicotrópicos y el desabasto de estos, por lo que las/los pacientes deben adquirirlos por su cuenta. Esta limitación se extiende a la atención en crisis, ya que no siempre se cuentan con los recursos para manejar episodios que requieren de medicamento urgente.

Aquí, [a los pacientes] les hace una clasificación socioeconómica [por parte de] trabajo social y el nivel que les ponen es un número que va al frente de su carné. Entonces, si es uno, dos o tres, tienen derecho a farmacia y se los regala [el medicamento]. Claro, 
hay un límite de cajas que se puede dar y entonces el problema es cuando no hay (op-18-M62).

Recursos humanos. Las/los participantes reconocieron que no se aborda el tema de las conductas suicidas en los cursos de salud mental durante su formación de pregrado. Por este motivo, afirman que su aprendizaje en el manejo a las conductas suicidas se da "sobre la marcha", a partir de la experiencia acumulada en la práctica o durante los estudios de posgrado. Aunque se capaciten en sus lugares de trabajo, la oferta educativa no cuenta con cursos específicos sobre las conductas suicidas.

[...] pues, creo yo que buscando personal especializado para [la atención de las conductas suicidas], gente preparada para esto, porque aquí realmente nos formamos en el camino. O sea, tú sales de la carrera y en el camino te vas formando y vas especializándote de acuerdo con tus inclinaciones, tus inquietudes (OP-13-M).

\section{Tema 4. Experiencias de los proveedores de servicios alrededor de las conductas suicidas}

Apoyo familiar en el proceso de atención. Uno de los principales retos que las personas participantes enfrentan en la atención es la sensibilización de familiares de las/los pacientes sobre las conductas suicidas. Aluden la dificultad de abordar los prejuicios que familiares expresan sobre las conductas suicidas y acerca del tratamiento psiquiátrico.

Además, los familiares tienen gran influencia en el apego terapéutico de las/los pacientes, ya que pueden apoyar el abandono de la medicación o de la terapia, al ver posibles signos de mejoría.

Me he dado cuenta de que sienten mejoría a los cuatro, cinco, seis meses y dejan un poquito de esas ideas y [los familiares] los dejan de traer, ya no les dan el medicamento. Como ya los ven bien, los dejan de traer, y entonces, los pacientes vuelven a reincidir un año o dos años después. Entonces, yo creo que lo más importante es que la familia los apoye hasta el último momento; aunque vean mejorías, continúen con el apoyo hasta que el médico o el especialista determine que ya puede ir disminuyendo (OP-11-M37).

Sobrecarga de trabajo y afectaciones personales. El proceso de atención de las personas con conductas suicidas fue descrito como "cansado", "triste", "pesado" y "difícil", lo cual permea el ánimo y la salud física del personal de salud.

A pesar de que el personal de salud debe lidiar diariamente con situaciones complejas, por la naturaleza de los servicios, no disponen de mecanismos de contención para liberar el estrés que su actividad les produce. Aunque son conscientes de la necesidad del autocuidado, no hay apoyos institucionales para que el personal de salud cuente con un proceso terapéutico.
Las/los participantes comentaron sentirse "frustrados" por no "poder hacer más" por las personas que acuden a la consulta con conductas suicidas.

Son pacientes [con conductas suicidas] que traen una gran carga emocional [...]. A veces, ya después de ver a un paciente, quisiera tirarme a dormir, y lo vale, pero requieren mucho tiempo, para asegurar, para garantizar que no van a lastimarse. Son pacientes que tienen mucho, requieren mucho compromiso (op-24-M47).

\section{Discusión}

En este estudio se evidenciaron diversas barreras para la atención de las conductas suicidas que experimentan las/ los prestadores de servicios de salud mental en dos centros de primer nivel ubicados en la Ciudad de México.

La falta de una estrategia nacional para la prevención del suicidio es la principal limitación que indicaron las personas participantes en el estudio, lo cual es un aspecto fundamental para guiar la atención. Al respecto, en Noruega se encontró que las/los profesionales de salud mental afirman que las guías nacionales permiten colocar la prevención del suicidio en la agenda de salud [28]; además, reflejan el compromiso de las autoridades con la problemática y facilitan la asignación de recursos [29]. En México, las normativas para la atención de las conductas suicidas se definen a nivel subnacional, lo que ha provocado la aplicación de intervenciones dispersas y sin presupuesto fijo, dificultando también la evaluación del impacto en salud de estas acciones [9,30].

En este estudio se constató que no hay un seguimiento de las/los pacientes después de la remisión a hospitales psiquiátricos. Aunque en México se desconocen las cifras del seguimiento de las/los pacientes con conductas suicidas, en países como Estados Unidos se estima que un tercio se les da seguimiento después del internamiento psiquiátrico [31]. A partir de un metaanálisis, se observó que las/los pacientes egresados de instituciones psiquiátricas tienen mayor riesgo de morir por suicidio en el corto plazo, en comparación con la población general [31].

Por otro lado, la atención de la salud mental durante la semana posterior al alta de los hospitales psiquiátricos se ha asociado con un menor riesgo de suicidio en comparación con 6 meses después del egreso. Además, la atención ambulatoria y el monitoreo previo a la atención hospitalaria se vincula con el correcto seguimiento de las/los pacientes [32]. En este sentido, es necesario reforzar la comunicación y los mecanismos de referencia y contrarreferencia entre los distintos niveles de atención.

Respecto a la disponibilidad de recursos, las/los profesionales de la salud nombraron la insuficiencia de insumos y de las unidades destinadas a la salud mental 
en México. A pesar de las recomendaciones internacionales para que la atención primaria sea el eje de atención de la salud mental, aún es necesario ampliar los establecimientos y la capacidad humana en este nivel [33]. Las/los participantes también señalaron la falta de entrenamiento para la atención de las conductas suicidas, tanto en su formación de pregrado como en sus centros de trabajo. En países de ingresos altos, como Estados Unidos, la proporción de quienes mencionan haber recibido entrenamiento para el abordaje de las conductas suicidas durante su formación de pregrado es menor del $50 \%$, [34], mientras que en países de ingresos medios y bajos no se tiene información puntual sobre este aspecto.

La atención de las/los pacientes con conductas suicidas requiere, por parte de las/los profesionales de la salud, de habilidades específicas, como el manejo de las propias emociones, el balance entre la relación con las/los pacientes y la distancia profesional, además de habilidades empíricas, como el reconocimiento de señales de alerta que indican algunas conductas suicidas o intención de autolesión de las/los pacientes [35]. La capacitación contribuye a mejorar la confianza de las/los profesionales para identificar, tratar y discutir las conductas suicidas con las/ los pacientes [34]. El efecto positivo se ha observado incluso en capacitaciones breves, de un día o menos, por lo que aun en escenarios de recursos limitados se puede implementar este tipo de acciones [34]. Sobre este punto, se ha demostrado que tanto la disponibilidad de servicios de salud mental como el tratamiento farmacológico y la psicoterapia son intervenciones efectivas para reducir las conductas suicidas a nivel poblacional [36].

Adicionalmente, las/los participantes mencionaron la carga emocional que representa atender a las/los pacientes con conductas suicidas, aunado a la falta que hay, en las instituciones, de programas de autocuidado dirigido al personal de salud.

La respuesta de las/los profesionales es similar a los hallazgos de estudios realizados en Nicaragua y Noruega, donde estos reportan malestar emocional posterior a la atención de un intento suicida, muchas veces asociado con un sentimiento de fracaso en la atención de las/los pacientes [35,37]. Se ha observado que compartir con colegas de trabajo la experiencia de la atención de las/ los pacientes con intento suicida ha sido de ayuda para las/los profesionales, junto con la capacitación para la atención de las conductas suicidas [38].

Asimismo, alrededor del $60 \%$ de las/los estudiantes de Psiquiatría y psiquiatras en México reportan algún problema de salud mental; entre los más frecuentemente mencionados están el desgaste profesional, la ansiedad y la depresión [39]. En países de ingresos altos, se ha encontrado mayor prevalencia de problemas de salud mental con las/los profesionales de salud mental que atienden a las/los pacientes con conductas suicidas, [40] por lo que es necesario que las estrategias de prevención de suicidio consideren el bienestar de estos profesionales de salud.

El presente estudio no está exento de limitaciones. El objetivo principal se centró en la experiencia del personal del primer nivel de atención; por tanto, no fue posible conocer la experiencia de las/los usuarios. Del mismo modo, la selección de las unidades de salud y la ciudad de estudio suponen condiciones únicas, así que los resultados no pueden ser generalizados a otras regiones del país. Sin embargo, la metodología empleada puede ser replicada en diversos contextos, con el fin de ampliar la comprensión del problema.

\section{Pasos por seguir}

La Secretaría de Salud propuso, a finales del 2020, la creación de la Comisión de Salud Mental y Adicciones, con el objetivo de unificar el manejo de la atención de la salud mental y las adicciones, además de implementar un modelo de atención de la salud mental basado en la atención primaria de salud integral [41]. La reforma incluye la creación de una estrategia nacional para la prevención del suicidio, que comprende la vigilancia epidemiológica, el diseño de intervenciones a nivel de atención primaria, la capacitación de diversos sectores y la investigación [41]. Por ende, es necesario evaluar, posteriormente, el efecto de estos cambios en el primer nivel de atención.

La población objetivo de este estudio se centró en personal del primer nivel de atención, por lo que se sugiere que futuras investigaciones abarquen la perspectiva de las personas que toman las decisiones, tanto en lo nacional como en lo local, del personal del segundo y tercer nivel de atención, y de las/los pacientes, quienes posiblemente enfrenten otros tipos de barreras que aquí no se identificaron.

Finalmente, esta investigación se desarrolló al inicio de la pandemia por COVID-19. Esta emergencia sanitaria ha puesto de relieve la necesidad de incluir y analizar los escenarios de emergencia en las políticas de prevención del suicidio, para continuar con la atención de la salud mental, y dar seguimiento a los datos epidemiológicos y estadísticos referentes a las conductas suicidas [42]. Por tanto, habrá de indagarse acerca de cómo la situación sanitaria en tiempo de pandemia afectó la salud mental de la población, las conductas suicidas y la respuesta de los servicios de salud.

\section{Conclusión}

Este estudio evidenció la ausencia de una institución rectora y programas a nivel nacional que orienten las acciones para la prevención y la atención de las conductas suicidas. Estos programas deben considerar las limitaciones del entorno y las características de los servicios 
de salud, así como las necesidades de las/los profesionales de salud. La falta de comunicación entre los niveles de atención apunta a la necesidad de integrar el sistema de salud mental.

Por otro lado, se requiere reforzar la capacitación y el entrenamiento del personal de salud mental en la detección y el tratamiento de las conductas suicidas, para mejorar la calidad de la atención, así como mejorar el sistema de referencia y contrarreferencia entre los distintos niveles de atención. Además, es indispensable proporcionar apoyo emocional al personal a cargo de estos servicios, para contender las situaciones que confrontan cada día en la atención.

\section{Agradecimientos}

Las autoras y el autor de este trabajo agradecen las facilidades prestadas en las dos unidades de salud para la realización de este estudio, así como al personal de salud que aceptó participar desinteresadamente.

\section{Financiación}

Este trabajo fue financiado por el Consejo Nacional de Ciencia y Tecnología de México, a través del programa de Becas Nacionales (\# 480539).

\section{Conflicto de interés}

Las investigadoras y el investigador declaran no tener conflicto de intereses en la presente investigación.

\section{Declaración de responsabilidad}

Lo expresado en el documento es responsabilidad de los autores y no de la Universidad de Antioquia ni de la $R e$ vista Facultad Nacional de Salud Pública.

\section{Declaración de autoría}

L. Gómez-García participó del diseño del estudio, análisis de datos, redacción del primer borrador, aprobación de la versión final. M. Agudelo-Botero, M. L. Arenas-Monreal, M. Rojas-Russell y R. Valdez-Santiago colaboraron en el diseño del estudio, el análisis y la interpretación de los datos, la revisión crítica y la aprobación del artículo. Todos los autores revisaron y aprobaron la versión final del artículo.

\section{Referencias}

1. Crosby A, Ortega L, Melanson C. Self-directed violence surveillance: Uniform definitions and recommended data elements, Version 1.0. Atlanta (GA): Centers for Disease Control and Prevention, National Center for Injury Prevention and Control; 2011.
2. Institute for Health Metrics and Evaluation (IHME). GBD Compare Seattle, wA [internet]; 2019 [citado 2021 may. 11]. Disponible en: http:/vizhub.healthdata.org/gbd-compare

3. Rivera-Rivera L, Fonseca-Pedrero E, Séris-Martínez M, et al. Prevalencia y factores psicológicos asociados con conducta suicida en adolescentes. Ensanut 2018-19. Salud Pública Mex. 2020;62(6):672-81. DOI: https://doi.org/10.21149/11555

4. Valdez-Santiago R, Hidalgo-Solorzano E, Mojarro-Iñiguez $\mathrm{M}$, et al. Attempted suicide among adolescents in Mexico: Prevalence and associated factors at the national level. Inj Prev. 2017;24(4):256-61. DOI: https://doi.org/10.1136/injuryprev-2016-042197

5. Robinson J, Bailey E, Witt $\mathrm{K}$, et al. What works in youth suicide prevention? A systematic review and meta-analysis. EClinicalMedicine. 2018;4-5:52-91. DoI: https://doi.org/10.1016/j. eclinm.2018.10.004

6. Díaz-Castro L, Cabello-Rangel H, Medina-Mora ME, et al. Necesidades de atención en salud mental y uso de servicios en población mexicana con trastornos mentales graves. Salud Pública Mex. 2019;62(1):72-79. DoI: https://doi.org/10.21149/10323

7. Martinez W, Galván J, et al. Barriers to integrating mental health services in community-based primary care settings in Mexico City: A qualitative analysis. Psychiatr Serv. 2017;68(5):497-502. DOI: https://doi.org/10.1176/appi.ps.201600141

8. Saavedra Solano N, Berenzon Gorn S, Galván Reyes J. Salud mental y atención primaria en México. Posibilidades y retos. Aten Primaria. 2016;48(4):258-64. DoI: https://doi.org/10.1016/j. aprim.2015.05.005

9. Valdez-Santiago R, Marín-Mendoza E, Gómez-García L, et al. Respuesta del sector salud a la conducta suicida de adolescentes en cinco estados de México: entre el desbordamiento y la precariedad. En: Valdez Santiago R, Arenas Monreal L, editores. Simplemente quería desaparecer... Aproximaciones a la conducta suicida de adolescentes en México. Cuernavaca, Morelos: Instituto Nacional de Salud Pública; 2019 [citado 2021 may. 4]. pp. 15-50. Disponible en: https://www.insp.mx/resources/images/stories/2019/Docs/190731_Conducta_suicida.pdf

10. Borges G, Wilcox HC, Medina-Mora ME, et al. Suicidal behavior in the Mexican National Comorbidity Survey (M-NCS): Lifetime and 12-month prevalence, psychiatric factors and service utilization. Salud Mental [internet]. 2005 [citado 2021 may. 4]; 28(2):40-47. Disponible en: http://www.revistasaludmental.mx/ index.php/salud_mental/article/view/1042/1040

11. Borges G, Orozco R, et al. Suicidio y conductas suicidas en México: retrospectiva y situación actual. Salud Pública Mex [internet]. 2010 [citado 2021 may. 4]; 52(4):292-304. Disponible en: https:// saludpublica.mx/index.php/spm/article/view/6979/8934

12. Romero-Martínez M, Shamah-Levy T, Cuevas-Nasu L, et al. Encuesta Nacional de Salud y Nutrición 2018. Cuestionario de Salud de Adolescentes (10 a 19 años) [base de datos electrónica]. México: Instituto Nacional de Estadística y Geografía e Instituto Nacional de Salud Pública. 2020 [citado 2021 may. 11]. Disponible en: https://ensanut.insp.mx/encuestas/ensanut2018/descargas.php

13. Hooper LM, Epstein SA, Weinfurt KP, et al. Predictors of primary care physicians' self-reported intention to conduct suicide risk assessments. J Behav Health Serv Res. 2012;39(2):103-15. DoI: https://doi.org/10.1007/s11414-011-9268-5

14. Valdez-Santiago R, Villalobos A, Arenas-Monreal L, et al. Comparative analysis of lifetime suicide attempts among Mexican adolescents, over the past 12 years. Int J Environ Res Public Health. 2021;18(10):1-13. DoI: https://doi.org/10.3390/ijerph18105419

15. Ibarra López AM, Sánchez Loyo LM, et al. Prevención y atención a los comportamientos suicidas: programas y estrategias de 
prevención del suicidio. En: Morfin López T, Ibarra López AM, editores. Fenómeno suicida: un acercamiento transdisciplinar. México: El Manual Moderno; 2015. pp. 29-45.

16. Valdez-Santiago R, Cruz-Bañares AL, et al. Living conditions of adolescents who have attempted suicide in Mexico. Int J Environ Res Public Health. 2020;17(16):1-12. DoI: https://doi. org/10.3390/ijerph 17165990

17. O’Brien BC, Harris IB, Beckman TJ, et al. Standards for reporting qualitative research: A synthesis of recommendations. Acad Med. 2014;89(9):1245-51. DoI: https://doi.org/10.1097/ ACM.0000000000000388

18. Donabedian A. Los espacios de la salud: aspectos fundamentales de la organización de la atención médica. México: Secretaría de Salud, Instituto Nacional de Salud Pública, Fondo de Cultura Económica; 1988.

19. Frenk J. El concepto y la medición de accesibilidad. Salud Pública Mex [internet]. 1985 [citado 2021 may. 4]; 27(5):438-5. Disponible en: https://saludpublica.mx/index.php/spm/article/view/422

20. Tanahashi T. Health service coverage and its evaluation. Bull World Health Organ [internet]. 1978 [citado 2021 may. 4]; 56(2):295-303. https://apps.who.int/iris/handle/10665/261736

21. Secretaría de Salud, Organización Panamericana de la Salud. Informe de la evaluación del sistema de salud mental en México utilizando el Instrumento de Evaluación para Sistemas de Salud Mental de la Organización Mundial de la Salud (IESM-OMS) [internet]. Secretaría de Salud; 2011 [citado 2021 may. 4]. Disponible en: https://www.who.int/mental_health/who_aims_country_reports/who_aims_report_mexico_es.pdf

22. Consejo Nacional de Evaluación de la Política de Desarrollo Social (CONEVAL). Medición de la pobreza. Pobreza municipal 2010-2015 [internet]. CONEVAL. 2015 [citado 2021 jul. 27]. Disponible en: https://www.coneval.org.mx/Medicion/Paginas/PobrezaInicio.aspx

23. Ritchie J, Spencer L, O'Connor W. Carrying out qualitative analysis. In: Ritchie J, Lewis J, editors. Qualitative research practice: A guide for social science students and researchers. London: Sage Publications; 2003. pp. 219-62.

24. Aday LA, Andersen R. A framework for the study of access to medical care. Health Serv Res[internet]. 1974 [citado 2021 may. 4]; 9(3):208-20. Disponible en: https://pubmed.ncbi.nlm.nih. gov/4436074/

25. Levesque JF, Harris MF, Russell G. Patient-centred access to health care: Conceptualising access at the interface of health systems and populations. Int J Equity Health. 2013;12:18. DoI: https://doi.org/10.1186/1475-9276-12-18

26. Penchansky R, Thomas JW. The concept of access: Definition and relationship to consumer satisfaction. Med Care. 1981;19(2):12740. DoI: https://doi.org/10.1097/00005650-198102000-00001

27. aTLAS.ti Scientific Software Development GmbH. aTLas.ti. Qualitative Data Analysis. 8.4.3 ed. Berlin; 2019.

28. Espeland K, Hjelmeland H, Loa Knizek B. A call for change from impersonal risk assessment to a relational approach: Professionals' reflections on the national guidelines for suicide prevention in mental health care in Norway. Int J Qual Stud Health Wellbeing. 2021;16(1):1868737. Dor: https://doi.org/10.1080/174826 31.2020 .1868737

29. Organización Panamericana de la Salud (OPS). Prevención del suicidio: un imperativo global. ops [internet]; 2014 [citado 2021 may. 11]. Disponible en: https://iris.paho.org/handle/10665.2/54141
30. Valdez-Santiago R, Marín-Mendoza E, Torres-Falcón M. Análisis comparativo del marco legal en salud mental y suicidio en México. Salud Pública Mex. 2021;63(4):554-64. Dor: https://doi. org $/ 10.21149 / 12310$

31. Chung D, Hadzi-Pavlovic D, Wang M, et al. Meta-analysis of suicide rates in the first week and the first month after psychiatric hospitalisation. BMJ Open. 2019;9(3):e023883. DOI: https://doi. org/10.1136/bmjopen-2018-023883

32. Fontanella CA, Warner LA, Steelesmith DL, et al. Association of timely outpatient mental health services for youths after psychiatric hospitalization with risk of death by suicide. JAMA Netw Open. 2020;3(8):e2012887. DoI: https://doi.org/10.1001/jamanetworkopen. 2020.12887

33. Berenzon Gorn S, Saavedra Solano N, Medina-Mora Icaza ME, et al. Evaluación del sistema de salud mental en México: ¿hacia dónde encaminar la atención? Rev Panam Salud Pública. 2013;33(4):252-8. DoI: https://doi.org/10.1590/s102049892013000400003

34. Wakai S, Schilling EA, Aseltine RH, et al. Suicide prevention skills, confidence and training: Results from the Zero Suicide Workforce Survey of behavioral health care professionals. SAGE Open Medicine. 2020;8:2050312120933152. DOI: https://doi. org/10.1177/2050312120933152

35. Hagen J, Knizek BL, Hjelmeland H. Mental health nurses' experiences of caring for suicidal patients in psychiatric wards: An emotional endeavor. Arch Psychiatr Nurs. 2017;31(1):31-37. Dor: https://doi.org/10.1016/j.apnu.2016.07.018

36. Zalsman G, Hawton K, Wasserman D, et al. Suicide prevention strategies revisited: 10-year systematic review. Lancet Psychiatry. 2016;3(7):646-59. DOI: https://doi.org/10.1016/S22150366(16)30030-X

37. Obando Medina C, Kullgren G, Dahlblom K. A qualitative study on primary health care professionals' perceptions of mental health, suicidal problems and help-seeking among young people in Nicaragua. BMC Fam Pract. 2014;15:129. DoI: https://doi. org/10.1186/1471-2296-15-129

38. Alhamidi SA, Alyousef SM. Perceptions of mental health nurses toward caring for suicidal hospital inpatients in Saudi Arabia. Death Stud. 2020:1-10. Dor: https://doi.org/10.1080/07481187.2 020.1801894

39. Fresán A, Guízar-Sánchez D, Yoldi-Negrete M, et al. Identifying risk factors for self-reported mental health problems in psychiatry trainees and psychiatrists in Mexico. Acad Psychiatry. 2021. DOI: https://doi.org/10.1007/s40596-021-01506-y

40. Johnson J, Hall LH, Berzins K, et al. Mental healthcare staff wellbeing and burnout: A narrative review of trends, causes, implications, and recommendations for future interventions. Int. J. Ment. Health Nurs. 2018;27(1):20-32. Dor: https://doi.org/10.1111/inm.12416

41. México, Secretaría de Salud. Programa de Acción Específico. Salud Mental y Adicciones 2020-2024. México: Secretaría de Salud [internet]; 2021 [citado 2021 jul. 27]. Disponible en: https://www. gob.mx/cms/uploads/attachment/file/626039/PAE_CONASAMA_26_3_21.pdf?fbclid=IwAR2ulj1-I3h-jgHjmDhlKopRwNitgSkhcmAZVEdh2Vc0WPcNw3UBh-i9T50

42. Ivbijaro G, Kolkiewicz L, Goldberg D, et al. Suicide prevention and COVID-19. Asia Pac Psychiatry. 2021;(13)3:e12482. DoI: https://doi.org/10.1111/appy.12482 